\title{
EFFECT OF EXOGENOUS ABSCISIC ACID ON ACCUMULATION OF RAFFINOSE FAMILY OLIGOSACCHARIDES AND GALACTOSYL CYCLITOLS IN TINY VETCH SEEDS (VICIA HIRSUTA [L.] S.F. GRAY)
}

\author{
LesŁaw B. LAHUTA ${ }^{1}$, RYSZARD J. GÓRECKI ${ }^{1}$, Ewa GOJŁO ${ }^{1}$, MARCin HorbOWiCZ ${ }^{2}$ \\ ${ }^{1}$ Department of Plant Physiology and Biotechnology, Warmia and Mazury University \\ Oczapowskiego 1A, 10-957 Olsztyn, Poland \\ e-mail: lahuta@uwm.edu.pl \\ ${ }^{2}$ Vegetable Quality Laboratory, Research Institute of Vegetable Crops \\ Konstytucji 3 Maja 1/3, 96-100 Skierniewice, Poland
}

(Received: March 26, 2004. Accepted: May 20, 2004)

\begin{abstract}
The role of the abscisic acid (ABA) in biosynthesis of raffinose family oligosaccharides (RFOs) and galactosyl cyclitols (Gal-C) in tiny vetch (Vicia hirsuta [L.] S.F. Gray) seeds was investigated. The ABA was applied through incubation of seed at various stage of its development. The level of RFOs and Gal-C was determined in seed maturing on plant and in seed maturing in vitro. In early stages of $V$. hirsuta seed development, the ABA activated the biosynthesis of galactinol, although the level of arisen galactinol quickly declined. In the later stages of $V$. hirsuta seed development ABA had stimulatory effect of RFOs and Gal-C biosynthesis. Influence of ABA on biosynthesis of $\alpha-$ -galactosides in Vicia hirsuta seed seems to be dependent on abscisic acid concentration. Low concentration of ABA had stimulatory effect on $\alpha$-galactosides biosynthesis, but high concentration of ABA inhibited the process.
\end{abstract}

KEY WORDS: ABA, raffinose, stachyose, verbascose, galactosyl cyclitols, Vicia hirsuta.

\section{INTRODUCTION}

Raffinose family oligosaccharides (RFOs) and $\alpha$-galactosides of cyclitols are widespread in legume seeds (Horbowicz and Obendorf 1994). Both types of carbohydrates have been implicated in the protection of seeds against damage during seed dehydration and aging, seed survival and storability (Obendorf 1997). They may protect membranes, proteins, and nucleic acids against the damage that occurs during and upon the withdrawal of water in the drying seeds. This protective role has been explained mainly by their capacity to retain the integrity of membranes through their interaction with the phospholipids, by replacing water during dehydration (Bentsink et al. 2000; Hincha et al. 2003). This is reason why RFOs appear during the late stages of seed development and desiccation process (Avigad and Dey 1997).

RFOs are galactosides of sucrose to which galactose units are added to the glucose moiety of sucrose through $\alpha-6$ bonds. The reactions are catalyzed by the enzymes raffinose synthase and stachyose synthase. RFOs have been extensively characterized as principle agents in carbon translocation in plants but have also been observed to accumulate under cold, drought or salinity stress, implying a role for RFOs in stress adaptation.
The first step in the biosynthesis of RFOs is the transfer of the galactosyl residue from the galactinol to sucrose. This reaction results in the formation of raffinose, the first member in the family, which serves as the acceptor molecule for the transfer of another galactosyl residue from galactinol, to form stachyose, and further to verbascose. Galactinol appears in seeds in phase of quick growth of its embryo and precedes accumulation of RFOs (Peterbauer et al. 1998; Peterbauer et al. 2001).

Desiccation of seeds out of plant causes biosynthesis and accumulation of both types of $\alpha$-galactosides, derivatives of sucrose and cyclitols and it is convenient method for studies of physiological processes during seed maturation and drying (Horbowicz et al. 1995; Górecki et al. 1997; Obendorf et al. 1998). During maturation of seed in ex planta the level of these carbohydrates depends on seed development stage and conditions of the experiment. High ambient temperature and drought stress during plant vegetation increase level of RFOs and $\alpha$-galactosides of cyclitols (Lahuta et al. 2000; Zalewski et al. 2001).

In vegetative tissues of Arabidopsis drought- and coldstress induces three stress-responsive genes responsible for galactinol biosynthesis (Taji et al. 2002). In effect, plants accumulate a large amount of raffinose and galactinol, but not stachyose. In bean seed, maturing under cold stress, an 
increased expression of galactinol synthase was found (Liu et al. 1998). Drought stress can induce expression of galactinol synthase in sprouting tomato seeds too, and activity of that enzyme is positively correlated to RFOs level (Downie et al. 2003; Handley et al. 1983). Similarity of the accumulation RFOs as a response on drought stress in seeds matured on plants and ex planta suggests that there is common factor which influences the RFOs biosynthesis. It was found that introduction of ABA to somatic embryos of alfalfa increased their tolerance to desiccation stress, as well as the raffinose and stachyose content (Sreedhar et al. 2002).

ABA reduces rate of metabolic processes in cells, and therefore can effects on high concentration of carbohydrates (Hoekstra et al. 2001). During seed development, ABA is known to initiate: embryo maturation, synthesis of storage reserves and late embryogenesis abundant proteins, and initiation of seed dormancy (Xiong and Zhu 2003).

In our studies an effect of exogenous ABA on accumulation of the galactinol, RFOs, and $\alpha$-galactosides of D-pinitol in maturing ex-planta seeds of Vicia hirsuta was investigated.

\section{MATERIALS AND METHODS}

\section{Plant material}

Plants of tiny vetch (Vicia hirsuta (L.) S.F. Gray) were grown in $1 \mathrm{dm}^{3}$ pots (two plants per pot) containing a mixture of soil and sand (4:1) in a greenhouse with a $14 \mathrm{~h}-$ $20^{\circ} \mathrm{C} / 10 \mathrm{~h}-16^{\circ} \mathrm{C}$ day/night cycle. The plants were illuminated by fluorescent lamps (light intensity was maintained at $190 \mu \mathrm{mol} \mathrm{m} \mathrm{m}^{-2} \mathrm{~s}^{-1}$ ), watered daily and fertilized weekly with NPK fertilizer $\left(3: 1: 1,0.5 \mathrm{~g} \mathrm{pot}^{-1}\right)$. Flowers at the first and second node were tagged when fully opened. Seeds were collected at intervals of 2-4 days from 6-8 to 36 days after flowering (DAF) from pods at the first and second node. Water content was determined after drying at $100^{\circ} \mathrm{C}$ to constant weight. Seeds of various age were taken to explanta experiments with exogenous ABA. Carbohydrate content was analyzed during maturation of seed on plant as well.

\section{Chemicals}

Standards of: D-pinitol D-chiro-inositol and d-ononitol were obtained from Fine Chemicals (New Zeland), galactinol - from Wako Pure Chemicals Industries, Ltd. (Japan), verbascose from Megazyme (Wicklow, Ireland). ABA (2-cis-4-trans abscisic acid), myo-inositol, glucose, fructose, sucrose, raffinose, stachyose, and xylitol were purchased from Sigma. Galactopinitol A, di-galacto-pinitol A (ciceritol), and tri-galactopinitol A were isolated and purified from Vicia villosa Roth. seeds as previously described (Szczeciński et al. 2000).

\section{Experiment 1 .}

\section{The effect of $A B A$ on carbohydrates accumulation} during early stages of development of tiny vetch seeds

To the experiment a young seeds at 6-8 DAF, at the late stage on embryo differentiation (seeds contained more than $85 \%$ water) were used. Seeds were rinsed in double distilled water and placed in Petrie dishes $(5 \mathrm{~cm}$ diameter, $30 \mathrm{se}-$ eds per treatment) in $5 \mathrm{~mL}$ of water containing sucrose (40 $\mathrm{mM})$, myo-inositol (10 mM), D-pinitol $(5 \mathrm{mM})$ and ABA at finally concentration 0 (control), 10 , and $50 \mu \mathrm{M} \cdot \mathrm{L}^{-1}$. Seeds harvested at 10-12 DAF (at the beginning of seed filling) were placed in $5 \mathrm{~mL}$ of water containing sucrose $(50 \mathrm{mM})$ and $\mathrm{ABA}$ at finally concentration 0 (control), 10, and 50 $\mu \mathrm{M} \cdot \mathrm{L}^{-1}$. Contents of RFOs and galactosyl cyclitols were determined after 2, 4 and 7 day of incubation. Before analysis seeds were precisely washed with distilled water in order to remove carbohydrates present in medium. Content and composition of carbohydrates in incubation medium was similar to their levels in young Vicia hirsuta seeds.

\section{Experiment 2 .}

The effect of $A B A$ on carbohydrates accumulation in late stages of development of tiny vetch seeds

Another samples of seeds were collected at 18 DAF, 24 DAF (stages of intensive embryo growth and dry mass accumulation), and $28 \mathrm{DAF}$ (maximum fresh weight, just before beginning of seed desiccation), and then rinsed in distilled water and placed in Petrie dishes in $5 \mathrm{~mL}$ of water containing sucrose $(50 \mathrm{mM})$ and $\mathrm{ABA}$ at finally concentration 0 (control), 200, and $400 \mu \mathrm{M} \cdot \mathrm{L}^{-1}$. Changes of soluble carbohydrates levels were analyzed in seeds after 2, 4 and 6 days of incubation. Due to very low level of myo-inositol and D-pinitol in this development stages if investigated seeds, the incubation medium contained sucrose and ABA only.

\section{Analysis of raffinose family oligosacharrides (RFOs) and galactosyl cyclitols (Gal-C)}

RFOs and Gal-C in incubated seeds and in seeds maturing on plant were analyzed by capillary gas chromatography (Górecki et al. 1997) in modification according to Peterbauer et al. (2001). Results were presented as means of three replicates \pm standard error.

\section{RESULTS}

Seeds of Vicia hirsuta have reached maximal fresh weight between 24 and 28 day after flowering (DAF), and maximal values of dry weight on 28-32 DAF (Fig. 1). During desiccation process (28-36 DAF) no substantial changes of dry weight level were observed. In early stages of Vicia hirsuta seed development major soluble carbohydrates were sucrose, myo-inositol and D-pinitol (data not shown). Level of sucrose was changed irregularly, and myo-inositol and D-pinitol have declined during incubation ex-planta. ABA had no influence on sucrose, myo-inositol and D-pinitol accumulation (data not shown).

Biosynthesis of galactinol started between 14 and 18 DAF and reached maximum level at 26 DAF (Fig. 2). Accumulation of galactinol proceeded accumulation of sucrose $\alpha$-galactosides (RFOs). At first started raffinose biosynthesis (18-22 DAF), then 4 and 10 days later, stachyose and verbascose. At the same time when raffinose (18 DAF) appeared the first member of cyclitol galactosides (Gal-C) group - galactopinitol A (GPA) was found. The content of GPA increased subsequently to full seed maturity. Higher homologues of GPA - di-galactopinitol A (ciceritol) and tri-galactopinitol A (TGPA) were accumulated during seed desiccation process mainly. During seed maturation the content of RFOs was always higher than Gal-C (Fig. 2).

Experiment 1. Fresh weight of seeds collected on 6-8 DAF increased from $5.59 \mathrm{mg}$ to $6.73 \mathrm{mg} \mathrm{seed}^{-1}$ after 2 days of in- 


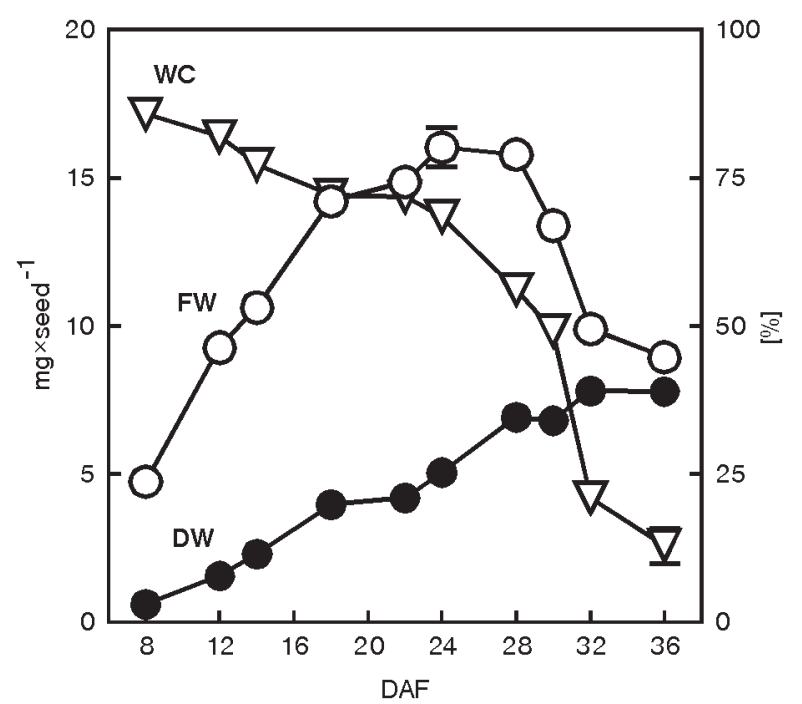

Fig. 1. Changes in fresh weight (FW), dry weight (DW) and water content (WC, \%) during maturation on planta of Vicia hirsuta seeds. Symbols represent the means \pm SE of five replications. Vertical bars represent \pm standard errors. Bars where not present fall within the symbols.

cubation. Longer process had no effect on seeds fresh weight accumulation (data not shown). After 2 days of seeds incubation in ABA contained medium the galactinol was found, but its level declined to traces after 4 days of the process.

There were no differences in the rate of galactinol biosynthesis in older seeds (collected on 10-12 DAF) incubated in medium contained 10 or $50 \mu \mathrm{M}$ of ABA. However, in contrary to young seeds (6-8 DAF), incubated older seeds (10-12 DAF) contained higher level of galactinol after 4 and 7 days of the process (Fig. 3). Raffinose family oligosaccharides and galactoside of D-pinitol were not detected in young seeds (6-12 DAF) incubated in ex-planta media contained the ABA or not.

Experiment 2. To the experiment seeds collected on 18 , 24 and 28 DAF were used. In these seeds accumulation of RFOs was initiated. Seeds harvested on 18 DAF contained galactinol and raffinose only, and in older (24 DAF) stachyose was found too. Seeds from 28 DAF contained all cyclitol galactosides, raffinose, stachyose and verbascose.

During incubation in control medium (contained of 50 $\mathrm{mM}$ of sucrose) accumulation of galactosyl cyclitols and
RFOs was observed, although seed age had a significant effect on the accumulation pattern of particular carbohydrates. During incubation of 18-days old seeds the level of galactinol was low and relatively stable (Fig. 4). In the same 18-days old seeds the raffinose, stachyose and verbascose were not accumulated in measurable levels (Fig. 5A, D, G).

In 24-days old seeds the content of galactinol increased gradually and after 6 days of incubation its level was 3-times higher than before the process. In 28-days old seeds the level of galactinol was three fold higher than in 24-days old, but incubation process did not change it. Biosynthesis and accumulation of galactinol in seeds of 18- and 24-days old during 6-days incubation was strongly stimulated by ABA, although on 6 day in 24-days old seeds no influence was found (Fig. 4). In older (28 DAF) seeds ABA did not change the accumulation pattern of galactinol, which was quite similar to control sample (Fig. 4).

During 6-days incubation of 18-days old seeds of $V$. hirsuta in control conditions (50 $\mathrm{mM}$ of sucrose) none of the RFOs was not found, but the presence of ABA have stimulatory effect on of all RFOs: raffinose, stachyose and verbascose biosynthesis (Fig. 5A, D, G). In older seeds (24 DAF) accumulation of RFOs started after 2 days of incubation either in medium contained of ABA or not (Fig. 5B, E, F). However, only high concentration of ABA $(400 \mu \mathrm{M})$ stimulated biosynthesis of raffinose and stachyose after 6 days of incubation, although had no effect on the accumulation of verbascose (Fig. 5B, E, F). In the case of older seeds (28 DAF) ABA delayed decline of raffinose level (Fig. 5C) and stimulated accumulation of stachyose and verbascose (Fig. 5F, I).

Similar to RFOs the presence of ABA (200 and $400 \mu \mathrm{M})$ have activated biosynthesis of galactosyl pinitol A in 18-days old seeds (Fig. 6A), but in the case od di-galactosyl pinitol A (ciceritol) only higher concentration of ABA had clear stimulatory effect after 6-days incubation process (Fig. 6D). In seeds from 24 and 28 DAF only ABA at concentration $200 \mu \mathrm{M}$ had no effect on the accumulation of GPA, ciceritol and TGPA in comparison to control conditions (Fig. 6B, C, E, F, H, I). Application of higher concentration of $\mathrm{ABA}(400 \mu \mathrm{M})$ caused an inhibitory effect on the biosynthesis of all investigated mono-, di- and tri-galactoside of D-pinitol (Fig. 6C, F, I). The phenomena is especially clear in the case of TGPA (Fig. 6I).
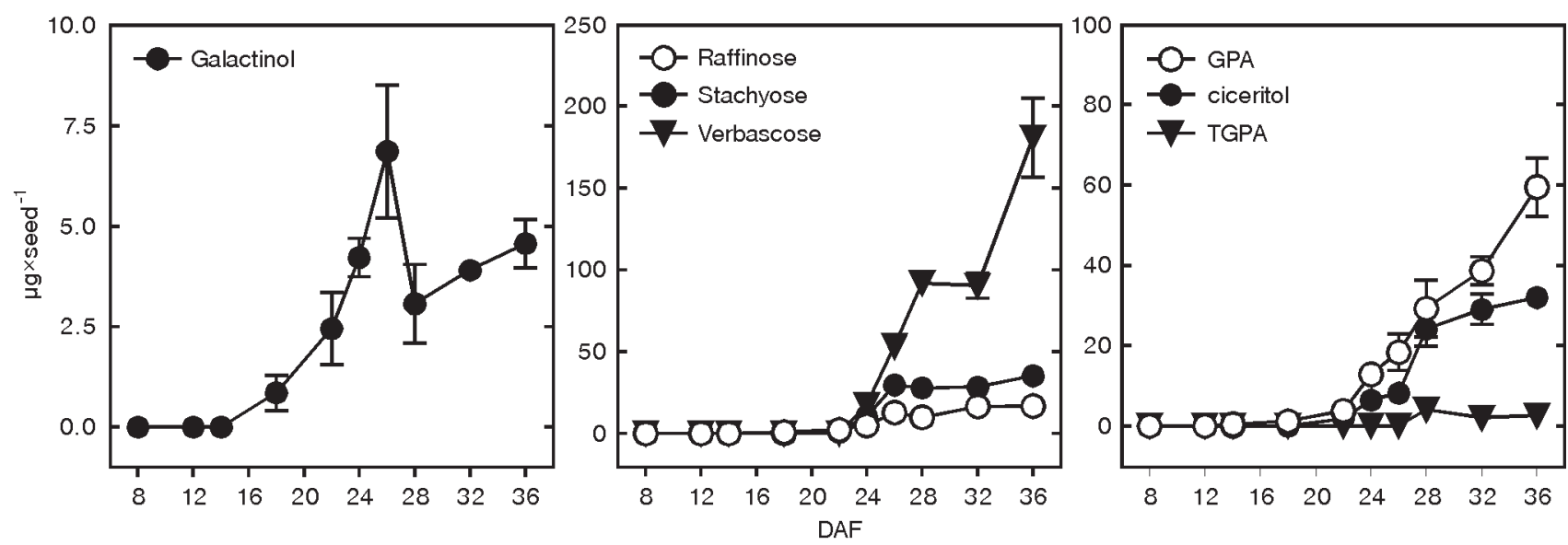

Fig. 2. Changes in galactinol, raffinose family oligosaccharides (RFOs), and galactosyl cyclitols (Gal-C) content during on planta maturation of Vicia hirsuta seeds. Symbols represent the means \pm SE of three replications. Vertical bars represent \pm standard errors. Bars where not present fall within the symbols. 


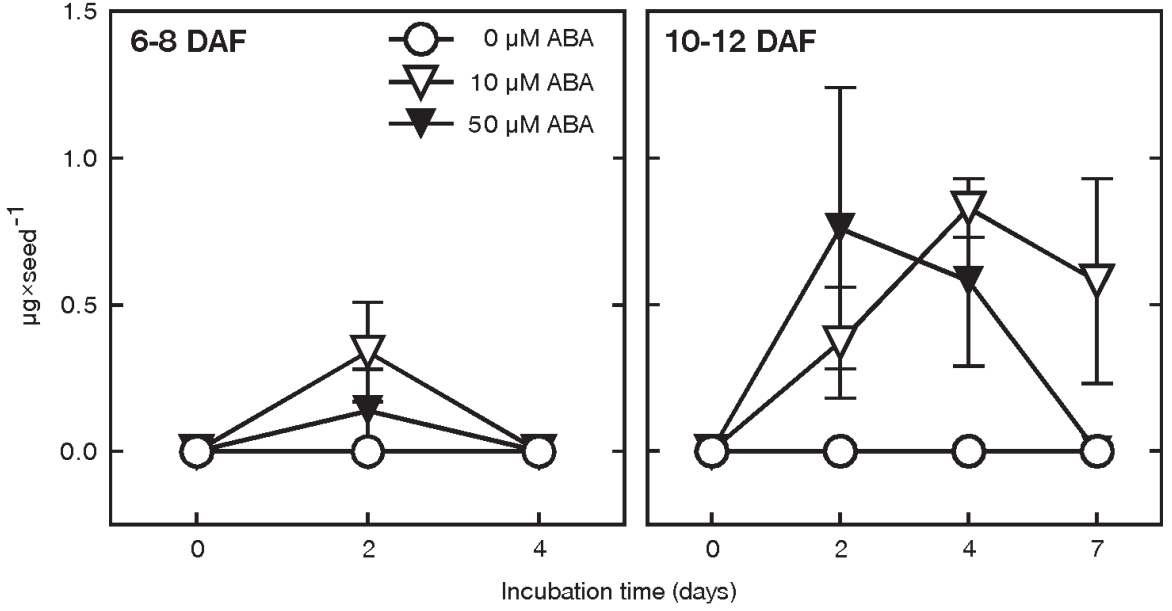

Fig. 3. The effect of ABA on the galactinol accumulation in Vicia hirsuta seeds of early development stages (6-8 DAF, and 10-12 DAF) incubated ex-planta. Vertical bars represent \pm standard errors. Bars where not present fall within the symbols.
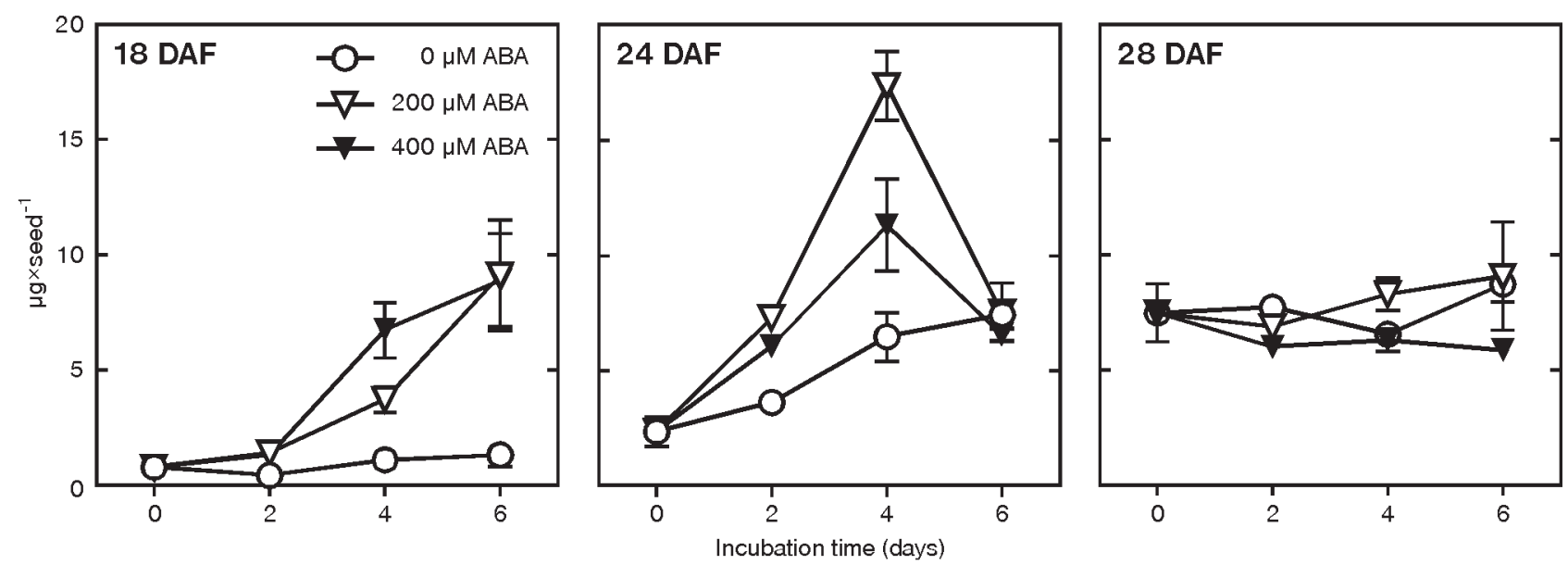

Fig. 4. The effect of ABA on the galactinol accumulation in Vicia hirsuta seeds of late development stages (18 DAF, 24 DAF, and 28 DAF) incubated explanta. Vertical bars represent \pm standard errors. Bars where not present fall within the symbols.

\section{DISCUSSION}

Raffinose family oligosaccharides (RFOs) and cyclitol galactosides accumulated during seed development are thought to play a role in the desiccation tolerance of seeds. However, the functions of RFOs in seed development and maturation have not been fully explained (Horbowicz and Obendorf 1994; Obendorf 1997).

Seed of tiny vetch (Vicia hirsuta) physiologically produces in high concentration of RFOs and cyclitol galactosides, during development and maturation on planta and in vitro. Biosynthesis of galactinol started between 14 and 18 DAF and reached maximum level at 28 DAF. Accumulation of galactinol proceeded accumulation of sucrose $\alpha$-galactosides (RFOs). At first started raffinose biosynthesis, then 4 and 10 days later, stachyose and verbascose. At the same time when raffinose appeared the first member of D-pinitol galactosides group - galactopinitol A (GPA) was found. The content of GPA increased subsequently to full seed maturity. Higher homologues of GPA - di-galactopinitol A (ciceritol) and tri-galactopinitol A were accumulated mainly during desiccation process. Intensification of biosynthesis of RFOs and cyclitol galactosides was accompanied by quick decline of galactinol level. Similar patterns of RFOs and cyclitol galactosides changes were found in developing seeds of other legumes: soybean, lupin and pea (Lowell and Kuo 1989; Modi et al. 2000; Górecki et al. 1997; Górecki et al. 2000; Peterbauer et al. 2001). Changes of galactinol level in legume seed was correlated with changes of galactinol synthase activity (Kuo et al. 1997; Peterbauer et al. 2001).

Carbohydrates have been postulated to help regulate the synthesis, conjugation and/or transport of phytohormones, such as abscisic acid. On the other hand, phytohormones can regulate sugar metabolism too (Gibson 2003). The abscisic acid inhibits the germination of many seeds, and in cereals ABA inhibits the expression of genes involved in storage reserve mobilization (Pritchard et al. 2002). In Arabidopsis seeds exogenous ABA caused that the stored lipid was broken down in sucrose, which content was twice higher in compare to normal growth conditions (Pritchard et al. 2002).

Non-matured seed transferred from plants to growing medium is exposed to stress. Stress is usually associated with increasing of endogenous ABA content. Action of ABA has often been followed by increased expression of some genes, similarly as during stress (Sauter et al. 2001; Vernieri et al. 2001; Zdunek and Lips 2001). Results obtained during our study shown that ABA can be involved in the regulation of biosynthesis of both types $\alpha$-galactosides: raffinose family oligosaccharides and galactosyl cyclitols in Vicia hirsuta seeds. Our investigations have shown that 


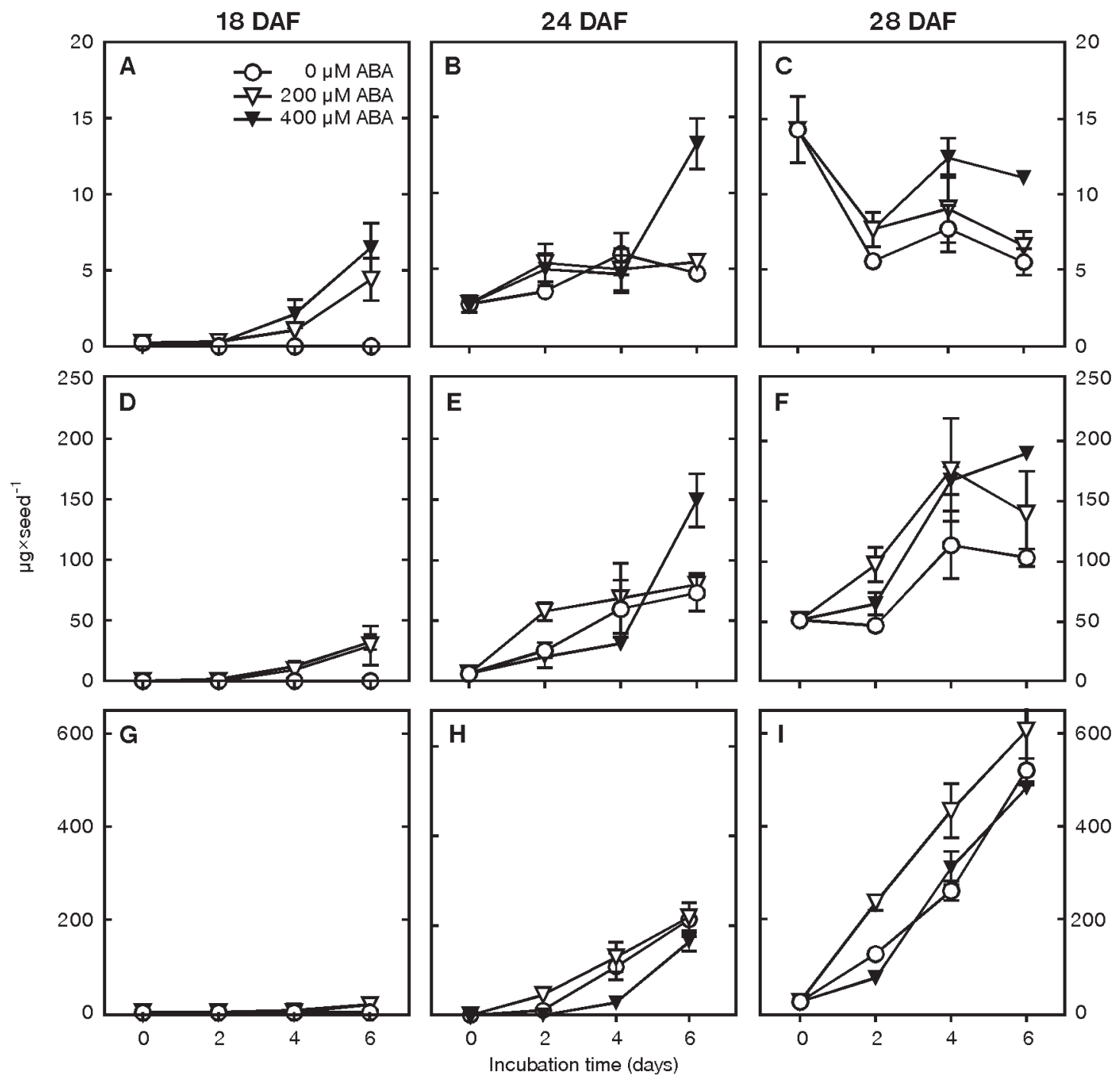

Fig. 5. The effect of ABA on the raffinose, stachyose and verbascose accumulation in Vicia hirsuta seeds of late development stages (18 DAF, 24 DAF, and 28 DAF) incubated ex-planta. Graphs A, B, C - raffinose; D, E, F - stachyose; G, H, I - verbascose. Vertical bars represent \pm standard errors. Bars where not present fall within the symbols.

the effect of ABA on accumulation pattern of galactinol, RFOs and Gal-C in $V$. hirsuta was dependent on seed age. The ABA have activated biosynthesis of galactinol in early stages of seed development (6-12 DAF), but the galactinol level quickly decreased. It seems like ABA stimulate genes and enzymes involved in biosynthesis of the compound. However, accelerated biosynthesis of galactinol did not influence of the RFOs accumulation in these stages of seed development.

We do not know what was endogenous level of $\mathrm{ABA}$ in that seed, but it can be quite possibly that in later stages of seed development concentration of endogenous ABA is enough for steering the accumulation of RFOs and galactosyl cyclitols. In early development stages of $V$. hirsuta seed concentration of the endogenous ABA is probably too low, and exogenously added hormone has an influence on accumulation of galactinol, and indirectly on both types $\alpha$-galactosides. Koornneef et al. (1989) have demonstrated that endogenous $\mathrm{ABA}$ is involved in the acquisition of desiccation tolerance in Arabidopsis thaliana seeds. By adding ABA to the culture medium at the appropriate stage of de- velopment, the dried somatic embryos retained its viability. The evidence found during our study can support their hypothesis.

Influence of $\mathrm{ABA}$ on $\alpha$-galactosides biosynthesis in $\mathrm{Vi}$ cia hirsuta seeds seems to be dependent on abscisic acid concentration too. ABA in low concentration had stimulatory effect on $\alpha$-galactosides biosynthesis, but high concentration inhibited the process. In Arabidopsis plants under drought, high-salinity and cold stresses galactinol and raffinose were accumulated in vegetative tissues (Taji et al. 2002). Authors have identified three stress-responsive genes of galactinol synthase ( GolS). Two of them were slightly induced by exogenous ABA treatment. Authors suggested, that galactinol and raffinose play a key function in drought tolerance in Arabidopsis plants. Although there is limited information on expression of GolS in developing seeds, it is possible that drought and other stresses can initiate its expression. In kidney bean seeds the GolS activity increases due to cold stress ( $\mathrm{Lin}$ et al. 1998). In germinating tomato seeds treated with exogenous ABA expression of LeGOLS-I was delayed (Downie et al. 2003). In radicles 
18 DAF
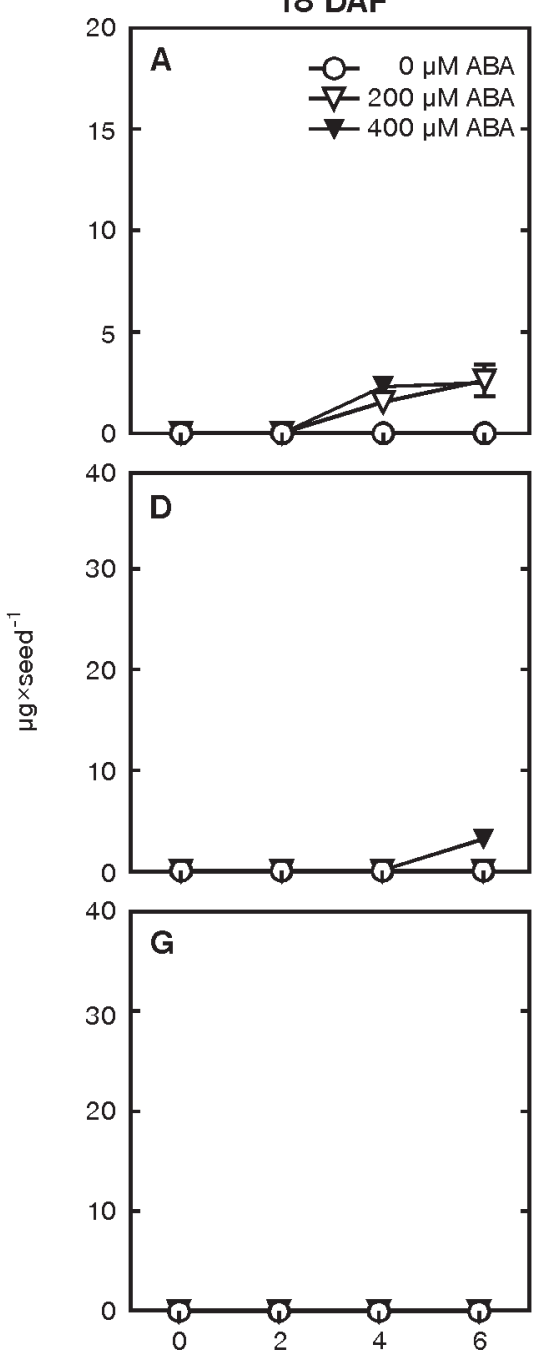

24 DAF
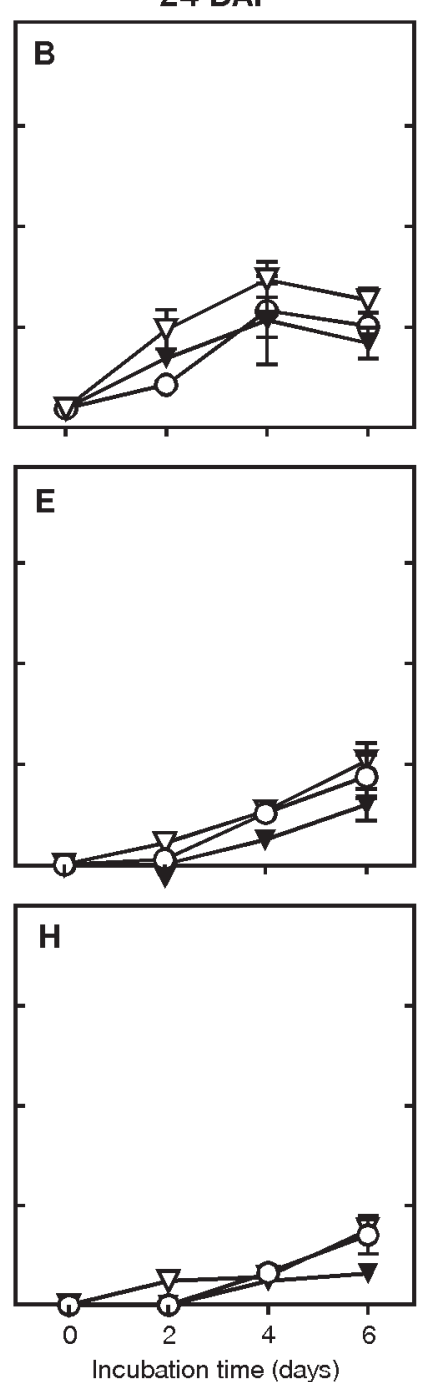

28 DAF
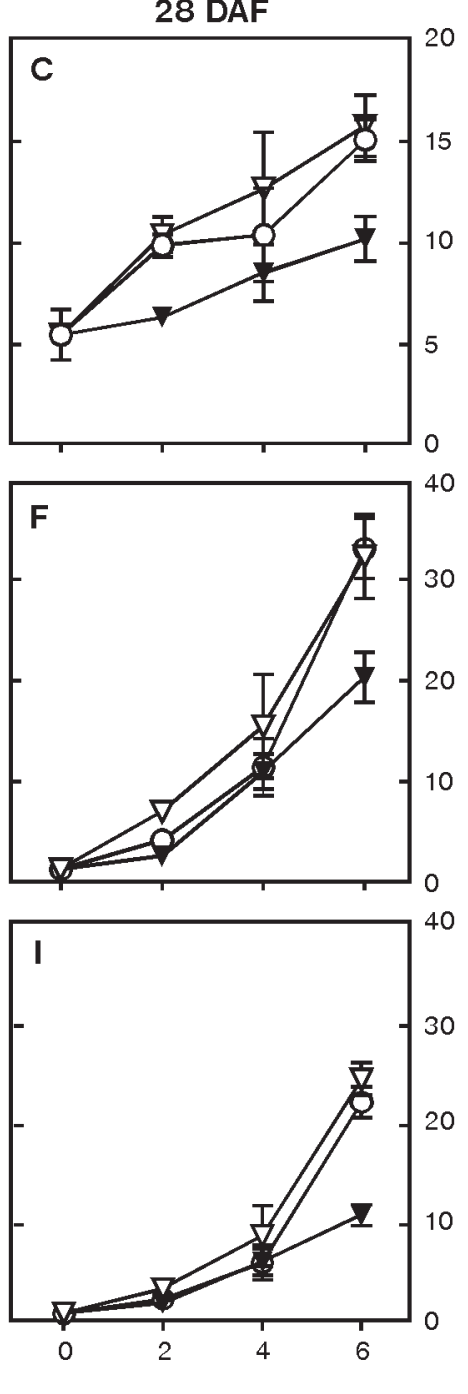

Fig. 6. The effect of ABA on the galactosyl cyclitols accumulation in Vicia hirsuta seeds of late development stages (18 DAF, 24 DAF, and 28 DAF) incubated ex-planta. Graphs: A, B, C - galactosyl pinitol A (GPA), D, E, F - di-galactosyl pinitol A (ciceritol, DGPA); G, H, I - tri-galactosyl pinitol A (TGPA). Vertical bars represent \pm standard errors. Bars where not present fall within the symbols.

of cucumber seeds imbibed with ABA, biosynthesis of the RFOs was enhanced, and after the seed drying the level of RFOs was two times higher (Lin et al. 1998).

According to our knowledge the presented by us data is the first evidence on the role of the ABA on biosynthesis of $\alpha$-galactosides of sucrose and galactosyl cyclitols in legume seed. The similarity of indirect effect of ABA on accumulation RFOs and galactosides of D-pinitol suggests that these carbohydrates may have a similar physiological role during seed maturation, and mechanisms of biosynthesis are common for many types of $\alpha$-galactosides, what supports earlier hypothesis of Peterbauer and Richter (2001). Data obtained in our study may be useful in understanding the role of RFOs, and galactosyl cyclitols and its relation with ABA, but the problem required further intensive studies.

\section{ACKNOWLEDGMENTS}

This work was supported by grant No 3P04C 03622 obtained from Ministry of Scientific Research and Information Technology of Poland.

\section{LITERATURE CITED}

AVIGAD G., DEY P.M. 1997. Carbohydrate metabolism: Storage carbohydrates. In: Dey P.M. Harbourne J. (eds) Plant biochemistry. San Diego; Academic Press, pp. 143-204

BENTSINK L., ALONSO-BLANCO C., VREUGDENHIL D., TESNIER K., GROOT S.P.C., KOORNNEEF M. 2000. Genetic analysis of seed-soluble oligosaccharides in relation to seed storability of Arabidopsis. Plant Physiol. 124: 1595-1604.

DOWNIE B., GURUSINGHE S., DAHAL P., THACKER R.R., SNYDER J.C., NONOGAKI H., YIM K., FUKANAGA K., ALVARADO V., BRADFORD K.J. 2003. Expression of a galactinol synthase gene in tomato seeds is up-regulated before maturation desiccation and again after imbibition whenever radicle protrusion is prevented. Plant Physiol. 131: 1347-1359.

GIBSON S.I. 2003. Sugar and phytohormone response pathways: navigating a signalling network. J. Exp. Bot. 55: 253-264.

GÓRECKI R.J., PIOTROWICZ-CIEŚLAK A.I., LAHUTA L.B., OBENDORF R.L. 1997. Soluble carbohydrates in desiccation tolerance of yellow lupin seeds during maturation and germination. Seed Sci. Res. 7: 107-115.

GÓRECKI R.J., LAHUTA L.B., JONES A.D., HEDLEY C.L. 2000. Soluble sugars in maturing pea seeds of different lines in relation to desiccation tolerance. In: M. Black, K.J. Brad- 
ford, J. Vasquez-Ramos (eds), Seed Biology: Advances and Applications. CAB International, Wallingford, UK, pp. 67-74.

HANDLEY L.W., PHARR D.M., MC FEETERS R.F. 1983. Relationship between galactinol synthase activity and sugar composition of leaves and seeds of several crop species. J. Am. Soc. Hortic. Sci. 108: 600-605.

HINCHA D.K., ZUTHER E., HEYER A.G. 2003. The preservation of liposomes by raffinose family oligosaccharides during drying is mediated by effects on fusion and lipid phase transitions. Biochim. Biophys Acta 1612: 172-177.

HORBOWICZ M., OBENDORF R.L. 1994. Seeds desiccation tolerance and storability: Dependence on flatulence-producing oligosaccharides and cyclitols - review and survey. Seed Sci. Res. 4: 385-405.

HORBOWICZ M., OBENDORF R.L., MC KERSIE B., VIANDS D.R. 1995. Soluble saccharides and cyclitols in alfalfa (Medicago sativa L.) somatic embryos, leaflets, and mature seeds. Plant Sci. 109: 191-198.

HOEKSTRA F.A., GOLOVINA E.A., BUITINK J. 2001. Mechanisms of plant desiccation tolerance. Trends Plant Sci. 6: 431-438.

KOORNNEEF M., HANHART C.J., HILHORST H.W.M., KARSSEN C.M. 1989. In vivo inhibition of seed development and reserve protein accumulation in recombinants of abscisic acid biosynthesis and responsiveness mutants in Arabidopsis thaliana. Plant Physiol. 90: 463-469.

KUO T.M., LOWELL C.A., SMITH P.T. 1997. Changes in soluble carbohydrates and enzymatic activities in maturing soybean seed tissues. Plant Sci. 125: 1-11.

LAHUTA L.B., ŁOGIN A., REJOWSKI A., SOCHA A., ZALEWSKI K. 2000. Influence of water deficit on the accumulation of sugars in developing field bean (Vicia faba var. minor) seeds. Seed Sci. Technol. 28: 93-100.

LOWELL C.A., KUO T.M. 1989. Oligosaccharide metabolism and accumulation in developing soybean seeds. Crop Sci. 29: 459-465.

LIN T.-P., YEN W.-L., CHIEN C.-T. 1998. Disappearance of desiccation tolerance of imbibed crop seeds is not associated with the decline of oligosaccharides. J. Exp. Bot. 49: 1203$-1212$.

LIU J.-J., KRENZ D.C., GALVEZ A.F., DE LUMEN B.O. 1998. Galactinol synthase (GS): increased enzyme activity and levels of mRNA due to cold and desiccation. Plant Sci. 134: 11-20.

MODI A.T., MC DONALD M.B., STREETER J.G. 2000. Soluble carbohydrates in soybean seeds during development and imbibition. Seed Sci. Technol. 28: 115-127.

OBENDORF R. 1997. Oligosaccharides and galactosyl cyclitols in seed desiccation tolerance. Seed Sci. Res. 7: 63-74.
OBENDORF R.L., HORBOWICZ M., DICKERMAN A.M., BRENAC P., SMITH M.E. 1998. Soluble oligosaccharides and galactosyl cyclitols in maturing soybean seeds in planta and in vitro. Crop Sci. 38: 78-84.

PETERBAUER T., RICHTER A. 2001. Biochemistry and physiology of raffinose family oligosaccharides and galactosyl cyclitols in seeds. Seed Sci. Res. 11: 185-197.

PETERBAUER T., PUSCHENREITER M., RICHTER A. 1998. Metabolism of galactosylononitol in seeds of Vigna umbellata. Plant Cell Physiol. 39: 334-341.

PETERBAUER T., LAHUTA L.B., BLÖCHL A., MUCHA J., JONES D.A., HEDLEY C.L., GÓRECKI R.J., RICHTER A. 2001. Analysis of the raffinose family oligosaccharide pathway in pea seeds with contrasting carbohydrate composition. Plant Physiol. 127: 1764-1772.

PRITCHARD L., CHARLTON W.L., BAKER A., GRAHAM I.A. 2002. Germination and storage reserve mobilization are regulated independently in Arabidopsis. Plant J. 31: 639-644.

SAUTER A., DAVIES W.J., HARTUNG W. 2001. The long-distance abscisic acid signal in the droughted plant: the fate of the hormone on its way from roots to shoot. J. Exp. Bot. 52: 1991-1997.

SREEDHAR L., WOLKERS W.F., HOEKSTRA F.A., BEWLEY J.D. 2002. In vivo characterization of the effects of abscisic acid and drying protocols associated with the acquisition of desiccation tolerance in alfalfa (Medicago sativa L.) somatic embryos. Ann. Bot. 89: 391-400.

SZCZECIŃSKI P., GRYFF-KELLER A., HORBOWICZ M., LAHUTA L.B. 2000. Galactosylpinitols isolated from vetch (Vicia villosa Roth.) seeds. J. Agric. Food Chem. 48: 2717-2720.

TAJI T., OHSUMI C., IUCHI S., SEKI M., KOBAYASHI M., YAMAGUCHI-SHINOZAKI K., SHINOZAKI K. 2002. Important roles of drought- and cold-inducible genes for galactinol synthase in stress tolerance in Arabidopsis thaliana. Plant J. 29: 417-426.

VERNIERI P., LENZI A., FIGARO M., TOGNONO F., PARDOSSI A. 2001. How the roots contribute to the ability of Phaseolus vulgaris L. to cope with chilling-induced water stress. J. Exp. Bot. 52: 2199-2206.

XIONG L., ZHU J.-K. 2003. Regulation of abscisic acid biosynthesis. Plant Physiol. 133: 29-36.

ZALEWSKI K., LAHUTA L.B., HORBOWICZ M. 2001. The effect of soil drought on the composition of carbohydrates in yellow lupin seeds and triticale kernels. Acta Physiol. Plant. 23: 73-78.

ZDUNEK E., LIPS S.H. 2001. Transport and accumulation rates of abscisic acid and aldehyde oxidase activity in Pisum sativum L. in response to suboptimal growth conditions. J. Exp. Bot. 52: 1269-1276. 